Trauma Berufskrankh 2006 8 [Suppl 2]: S192-S194 DOI 10.1007/s10039-005-1014-x

Online publiziert: 24. Mai 2005

(c) Springer Medizin Verlag 2005

K. J. Kraft

Hauptverband der gewerblichen Berufsgenossenschaften e.V., Sankt Augustin

\title{
Technische Voraussetzungen für DALE-UV
}

mationen unter http://www.kvno.de und http://www.initiative-praxis-edv.de). Die entsprechenden Spezifikationen der zu übermittelnden Feldinhalte sind in den Technischen Anlagen beschrieben und können unter http://www.dale-uv.de abgerufen werden. Dort werden auch die jeweils aktuellen Listen der Softwarehersteller, die in ihren Produkten über die entsprechenden Funktionen verfügen, veröffentlicht.

Für die elektronische Übermittlung freigegebene Formtexte der gesetzlichen Unfallversicherung sind in $\mathbf{A b b} \mathbf{1}$ und 2 dargestellt. Die Übermittlung der Ergänzungsberichte ist spätestens ab 01.07.2005 vorgesehen.

Die Formtextdaten werden erfasst. Der Erfassungsvorgang ist abgeschlossen, wenn eine Strukturprüfung innerhalb der Praxis-/Krankenhaussoftware erfolgt ist. Ziel dieser zwingend vorgeschriebenen Prüfung vor Ort ist die Vermeidung von unnötigen Datenübertragungskosten. Hierzu stellt der HVBG kostenlos ein Strukturprüfmodul in der jeweils gültigen Version zur Verfügung. Nach der vollständigen Erfassung wird das Dokument mit einer elektronischen Signatur versehen. Das nun fertige Dokument wird für den elektronischen Versand verschlüsselt und an eine Universelle Daten- annahme und Verteilstelle (UNI-DAV) der gesetzlichen Unfallversicherungsträger übermittelt. Diese unterhalten 2 UNIDAV. Eine ist beim HVBG installiert, die auch im Dienstleistungsverfahren die Aufgaben des Bundesverbandes der Unfallkassen (BUK) übernimmt. Die andere wird vom Bundesverband der landwirtschaftlichen BG (BLB) betrieben. Durch die Formulareingabe des empfangenden Unfallversicherungsträgers erfolgt automatisch die Zuordnung der jeweiligen UNI-DAV durch die Praxis-Krankenhaus-Softwaresysteme.

Der Versand der Belegkopien an die gesetzliche Krankenversicherung und ggf. an den weiterbehandelnden Arzt durch den D- oder H-Arzt entfällt. Die Kopien werden durch die UNI-DAV auf dem Postweg versandt. Zurzeit werden Gespräche mit den Trägern der gesetzlichen Krankenversicherung über den elektronischen Versand geführt. Der elektronische Versand zum weiterbehandelnden Arzt ist nach der Einführung der HPC geplant.

Die Weiterleitung der elektronischen Geschäftsvorfälle an den empfangenden Unfallversicherungsträger erfolgt zeitnah, in der Regel innerhalb einer Stunde.

Leider muss erwähnt werden, dass die Umsetzung des berufsgenossenschaftlichen Formularwesens in die Software- 
produkte der Praxis-/Krankenhaussysteme nicht in dem seitens der UV-Träger gewünschten und $z$. T. auch von den Herstellern zugesagten Umfang durchgeführt wurde. Während die Situation bei den niedergelassenen $\mathrm{D}$ - und $\mathrm{H}$-Ärzten noch gut ist, sind im Krankenhausbereich bisher wenige Ärzte in der Lage, an DALE-UV teilzunehmen.

\section{Anmeldverfahren}

Anmeldeformulare für die Teilnahme am DALE-UV-Verfahren sind bei den jeweiligen Landesverbänden zu erhalten oder aus unserer jeweils aktuellen Informationsbroschüre, die unter http://www.daleuv.de/download abrufbar ist. Das ausgefüllte Formular wird an die aufgedruckte Adresse beim HVBG gesandt. Dieser schickt dem Leistungserbringer eine Bestätigung. Eine Kopie der Anmeldung wird an die Zentralstelle des jeweiligen Softwarehauses versandt. Im Regelfall wird dieses seinen jeweiligen Service- oder Vertriebspartner informieren, der die weiteren Maßnahmen einleitet.

Es wurde festgelegt, dass die Unfallversicherungsträger ausdrücklich keinen Einfluss in die bestehende Kunden- (Arzt) und Lieferantenbeziehung (Softwarehersteller) nehmen. Vom Softwarehaus werden jedoch eine Statusüberwachung und eine Statusmeldung an den HVBG erwartet.

Für die Teilnahme an DALE-UV werden folgende Komponenten benötigt:

- ggf. Chipkartenleser und Smartcard

- Zugangspaket eines Providers für den sicheren Internetzugang

- Installation vorgenannter Komponenten

- evtl. Schulung/Einweisung in die DALE-UV-Abläufe

\section{Kosten, Kostenerstattung}

Für die Installation der erforderlichen Komponenten und evtl. neue Softwareversionen werden die Kosten durch den Service- und Vertriebspartner veranschlagt und abgerechnet. Die erforderliche, gesicherte Verbindung für die Datenkommunikation stellt ein Provider zur Verfü-

Trauma Berufskrankh 2006 : 8[Suppl 2]: S192-S194

DOI 10.1007/s10039-005-1014-x

○) Springer Medizin Verlag 2005

\section{K. J. Kraft}

\section{Technische Voraussetzungen für DALE-UV}

\section{Zusammenfassung}

Voraussetzung für die Teilnahme an DALEUV ist, dass beim Leistungserbringer die Formtexte der gesetzlichen Unfallversicherung in der Praxis-/Krankenhaussoftware integriert sind und dass die zu übermittelnden Geschäftsvorfälle elektronisch signiert und verschlüsselt versandt werden können. Zudem werden benötigt: Chipkartenleser und Smartcard, Zugangspaket eines Providers für den sicheren Internetzugang, Installation vorgenannter Komponenten und evtl. Schulung/Einweisung in die DALE-UVAbläufe. Anmeldeformulare für die Teilnahme am DALE-UV-Verfahren sind bei den jeweiligen Landesverbänden oder unter

\section{Requirements for DALE UV}

\section{Abstract}

Before a service provider can take part in DALE UV it is essential for the standard texts used in legally required accident insurance to be integrated in the software used in the practice/hospital providing the services and for electronic signatures and coding of the business events to be communicated to be possible. Other requirements are: a chipcard reader and a smartcard, access package of a provider for secure internet access, installation of the afore mentioned components, and training/tutoring in the DALE UV procedures. The necessary forms for applications to take part in the DALE UV procedure can be obtained from the appropriate asso- http://www.dale-uv.de/download zu erhalten. Für erforderliche Neuanschaffungen gibt es eine einmalige Bezuschussung, befristet zum 31.12.2005. Außerdem werden Übertragungsgebühren mit 0,35 EUR erstattet. Die Zusammenarbeit der D- und HÄrzte mit Abrechnungsstellen wird durch DALE-UV nicht beeinflusst.

\section{Schlüsselwörter}

Formtexte der gesetzlichen

Unfallversicherung · Software .

Chipkartenleser und Smartcard .

Anmeldeformulare $\cdot$ Bezuschussung

ciations in each land or downloaded from http://www.dale-uv.de/download. Until 31.12.2005 a grant, payable on one occasion only, is available for new equipment needed specifically for this purpose. In addition, transmission fees are reimbursed at a rate of 0,35 EUR. The way emergency doctors on call work with the accounts clearing houses will not be affected by DALE UV.

\section{Keywords}

Standard texts for legally required accident insurance - Software - Chipcard reader and smartcard · Application forms .

Grant assistance 
DALE - UV

\section{- D-Arzt-Verfahren, Formtexte}

7. Durchgangsarzt (D-Arzt) zum

$$
\text { UV-Träger }
$$

$\Rightarrow$ Durchgangsarztbericht F 1000 - früher D-13

$\Rightarrow$ Nachschaubericht F 2106 - früher D9a

$\Rightarrow$ Meldung F 2222 - früher KD-10

$\Rightarrow$ Zwischenbericht F 2100 - früher AV 5

$\Rightarrow$ Rechnungsvordrucke F 9990, F 9992, F 9994 - früher R1-R3

$\rightleftarrows$ Ergänzungsberichte F1002, F1004, F1006, F1008

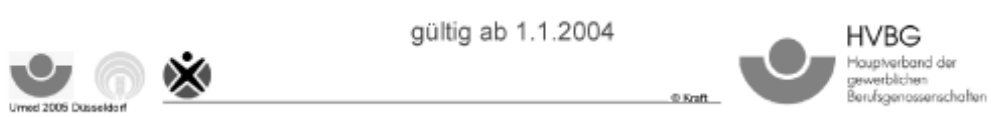

Abb. $1 \Delta$ Formtexte für das D-Arzt-Verfahren

DALE - UV

\section{- Formtexte H-Arzt-Verfahren}

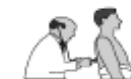

Datenfluss vom H-Arzt zum UV-Träger

C H-Arzt-Bericht F1020 (früher H13)

$\Rightarrow$ Verlaufsbericht (F2108)

$\Rightarrow$ Meldung F 2222 - früher KD-10

$\Rightarrow$ Rechnungsvordrucke F 9990, F 9992, F 9994

- früher R1-R3

$\Rightarrow$ Ergänzungsberichte F 1002, F 1004, F 1006, F 1008

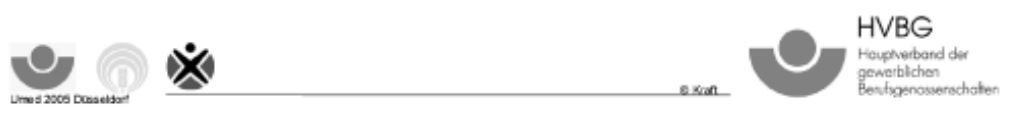

Abb. $2 \Delta$ Formtexte für das H-Arzt-Verfahren

gung, der diese Leistung auch abrechnet. Die UV-Träger erstatten für diese Kosten einen einmaligen Zuschuss von 200 EUR. Die Überweisung erfolgt automatisch durch den HVBG (UNI-DAV) nach Eingang der ersten Geschäftsvorfälle über DALE-UV. Die Bezuschussung gilt nur bis 31.12.2005.

Außerdem berechnet der Provider Verbindungsgebühren für die Transaktionen. Diese werden bei den Berichten, für die bisher Porto abgerechnet wurde, mit o,35 EUR erstattet. Damit sind die Kosten für die oben angeführte Datenübermittlung ab 150 D-Berichten/Jahr gedeckt.

\section{Abrechnungsstellen}

Die Zusammenarbeit der D- und H-Ärzte mit Abrechnungsstellen (z. B. PVS) wird durch DALE-UV nicht beeinflusst. Wie bisher werden die Leistungen von den
Abrechnungsstellen mit den UV-Trägern abgerechnet. Einige Abrechnungsstellen übermitteln ihre Rechnungen bereits elektronisch.

\section{Projektleitung und Support}

Projektleitung: Dr. J. Gebhard, Tel.: 02241-2311444,

E-Mail: projektleitung@dale-uv.de Support: C. Kreiß, Tel.: 02241-231133O, E-Mail: support@dale-uv.de

\section{Korrespondierender Autor K. J. Kraft}

Hauptverband der gewerblichen Berufsgenossenschaften e.V., Alte Heerstraße 111, 53757 Sankt Augustin E-Mail: Klaus-Juergen.Kraft@HVBG.de

Interessenkonflikt: Der korrespondierende Autor versichert, dass keine Verbindungen mit einer Firma, deren Produkt in dem Artikel genannt ist, oder einer Firma, die ein Konkurrenzprodukt vertreibt, bestehen. 